\title{
Increased Risk of Valvular Heart Disease in Systemic Sclerosis: An Underrecognized Cardiac Complication
}

\author{
Reto D. Kurmann ${ }^{1}$ (D), Edward A. El-Am² (i), Yasser A. Radwan ${ }^{3}$ (i), Avneek S. Sandhu', \\ Cynthia S. Crowson ${ }^{5}$ (D) Eric L. Matteson ${ }^{5}$ (D), Kenneth J. Warrington ${ }^{3}$ (D), Rekha Mankad ${ }^{6}$ (D), \\ and Ashima $\mathrm{Makol}^{3}$ (i)
}

\begin{abstract}
Objective. Cardiac involvement is a poor prognostic marker in systemic sclerosis (SSc). While diastolic dysfunction, myocardial fibrosis, and arrhythmias are traditionally considered features of primary cardiac involvement in SSc, the incidence of valvular heart disease (VHD) is not well reported. Our objective was to examine the prevalence of VHD at the time of SSc diagnosis and incidence of VHD during follow-up compared to non-SSc subjects.
\end{abstract}

Methods. Medical records of patients with suspicion of SSc were reviewed to identify incident cases. SSc subjects were matched $1: 2$ by age and sex to non-SSc subjects.

Results. The study included 78 incident SSc cases and 156 non-SSc comparators (56 yrs [ \pm 15.7$], 91 \%$ female). A nearly 4 -fold increase in the prevalence of moderate/severe VHD prior to SSc diagnosis compared to non-SSc subjects ( $6 \%$ vs $0 \% ; P=0.004$ ) was identified. During follow-up, 18 SSc and 12 non-SSc patients developed moderate/severe VHD. The cumulative incidence of VHD at 10 years after SSc incidence/index was $17.9 \%$ (95\% CI 10.7-29.9) in patients with SSc compared with $2.3 \%$ (95\% CI 0.7-7.0) in non-SSc subjects (HR 4.23, 95\% CI 2.03-8.83). Coronary artery disease was the only significant risk factor for VHD. Conclusion. Patients with SSc have a 4 -fold increase in the prevalence of moderate/severe VHD at diagnosis compared to non-SSc patients. They also have a 4-fold increased risk of developing moderate/severe VHD after diagnosis of SSc. Aortic stenosis and mitral regurgitation have a much higher prevalence in patients with SSc, besides secondary tricuspid regurgitation. Underlying mechanisms for this association require further elucidation.

Key Indexing Terms: cardiomyopathies, heart disease, incidence, scleroderma, systemic sclerosis, valvular heart disease

Systemic sclerosis (SSc) is a complex, heterogeneous autoimmune connective tissue disease characterized by microvascular injury, extracellular matrix deposition, and widespread fibrosis of numerous tissues and organs, particularly lungs and heart. ${ }^{1,2,3}$ Cardiac involvement in SSc is common, with reported prevalence in the range of $15-35 \% .{ }^{4,5,6,7}$ Cardiac involvement is likely underestimated and underrecognized until late in the disease course since most manifestations remain subclinical and occult in nature. Subclinical cardiac involvement has been reported in

This study was made possible using the resources of the Rochester Epidemiology Project, which is supported by the National Institute on Aging of the National Institutes of Health (NIH) under Award Number R01AG034676, and Grant Number UL1 TR000135 from the National Center for Advancing Translational Sciences (NCATS), one of the institutes of the NIH. The study was also supported by the John M. Nasseff, Sr. Clinician Career Development Award in Rheumatology to AM and Mayo Clinic CTSA through grant number UL1 TR002377 from the NCATS. The content is solely the responsibility of the authors and does not necessarily represent the official views of the NIH. RDK received funding from Research Funds of the Department of Rheumatology and Clinical Immunology, University of Bern, Switzerland.

${ }^{I}$ R.D. Kurmann, MD, Department of Cardiovascular Diseases, Mayo Clinic, Rochester, Minnesota, USA, and Department of Cardiology, Heart Center Lucerne Hospital, Lucerne, Switzerland; ' 2 .A. El-Am, MD, Department of Cardiovascular Diseases, Mayo Clinic, Rochester, Minnesota, and approximately $70 \%$ of patients depending on the degree of suspicion and screening tools utilized. ${ }^{4,5,6,7}$ Involvement of the heart is a strong predictor of mortality in SSc and is associated with a poor prognosis, with up to $70 \%$ mortality reported at 5 years. ${ }^{4,5}$ Therefore, early detection and monitoring of cardiac conditions are important aspects of management in this patient population. All anatomic domains of the heart can be affected by the fibrotic and vascular processes of SSc. ${ }^{5}$ In addition, cardiac involvement can occur secondary to interstitial lung disease (ILD), pulmonary

Department of Medicine, Indiana University School of Medicine, Indianapolis, Indiana, USA ${ }^{3} Y . A$. Radwan, MD, K.J. Warrington, MD, A. Makol, MD, Division of Rheumatology, Mayo Clinic, Rochester, Minnesota, USA; ${ }^{4} A . S$. Sandhu, MD, Department of Medicine, Kettering Health Medical Center, Dayton, Ohio, USA; ${ }^{5}$ C.S. Crowson, PhD, E.L. Matteson, MD, MPH, Division of Rheumatology, and Department of Health Sciences Research, Mayo Clinic, Rochester, Minnesota, USA; ${ }^{6}$. Mankad, MD, Department of Cardiovascular Diseases, Mayo Clinic, Rochester, Minnesota, USA.

The authors declare no conflicts of interest.

Address correspondence to Dr. A. Makol, Associate Professor of Medicine, Division of Rheumatology, Mayo Clinic, Rochester, MN 55905, USA. Email:makol.ashima@mayo.edu.

Open Access Article. For details see Reprints and Permissions at jrheum.org. Accepted for publication December 17, 2020. 
arterial hypertension (PAH), scleroderma renal crisis (SRC), or by medications used to treat SSc. ${ }^{4,5,6}$ Most studies have looked at pericarditis, myocarditis, heart failure, diastolic dysfunction, arrhythmias, and conduction disturbances. ${ }^{45,6,7}$ However, little is known about the occurrence of valvular heart disease (VHD) in SSc. Therefore, the purpose of our study was to investigate the prevalence of VHD at time of SSc diagnosis and the incidence of VHD during follow-up compared to non-SSc subjects in a population-based cohort.

\section{METHODS}

The Mayo Clinic and Olmsted Medical Center Institutional Review Boards approved this study (IRB 17-005603). In this retrospective study, we reviewed all adults (age $\geq 18$ yrs) with a diagnosis or suspicion of SSc among residents of Olmsted County, Minnesota, from January 1, 1980, to December 31, 2016, to identify incident cases of SSc. Patient consent for publication was not required.

The diagnosis of SSc was established based on physician diagnosis, and fulfillment of 2013 criteria for SSc was ascertained. ${ }^{8}$ Clinical data were gathered by using the Rochester Epidemiology Project (REP), a medical record system linking together all the medical records of Olmsted County residents from multiple healthcare providers and institutions. ${ }^{9}$ Patients with other rheumatologic diseases were excluded from the study. A comparison cohort was randomly selected from the same population of Olmsted County residents. The SSc and comparator patients were matched 1:2 by age ( \pm 3 yrs) and sex. Index/incidence date was based on date of physician diagnosis of SSc and/or date of appearance of any additional non-Raynaud symptoms.

Data about clinical characteristics and cardiovascular (CV) risk factors, including smoking status, obesity, hypertension, dyslipidemia, diabetes mellitus, use of aspirin, known coronary heart disease, peripheral artery disease, abdominal aneurysm, atrial fibrillation, and heart failure, were manually extracted from the medical records and based on physician diagnosis. Antihypertensive medications alone were not counted as a diagnosis of hypertension in patients with SSc due to the increased prevalence of vasodilator use in this population of patients. Renal involvement was defined as SRC, whereas gastrointestinal (GI) involvement was defined GI dysmotility or pseudo-obstruction or gastric antral vascular ectasia or small intestinal bacterial overgrowth. ILD diagnosis was based on computed tomography. PAH diagnosis was based on right heart catheterization (RHC) or echocardiogram criteria. Definitive PAH diagnosis was based on RHC as the gold standard for diagnosis with mean pulmonary artery pressure $>20 \mathrm{mmHg}$, pulmonary vascular resistance $\geq 3$ Wood units, and pulmonary arterial wedge pressure $\leq 15 \mathrm{mmHg}$. Probable PAH diagnosis was based on echocardiogram for patients who did not have RHC criteria, and included both right ventricular systolic pressure $>45 \mathrm{mmHg}$ and tricuspid regurgitant velocity $>3.4 \mathrm{~m} / \mathrm{s}$. Echocardiogram and RHC reports from January 1, 1980, to December 31, 2018, were manually reviewed to determine the occurrence of any VHD including aortic, mitral, pulmonary, or tricuspid valve stenosis and/or regurgitation as defined by the 2014 American Heart Association/American College of Cardiology guidelines. ${ }^{10}$

Descriptive statistics (e.g., percentages, mean) were used to summarize patient characteristics for each cohort. Baseline comparisons between cohorts were performed using chi-square and rank-sum tests. The analyses of VHD were performed using all patients and in the subset of patients who received echocardiograms. The prevalence of prior VHD overall and by type was compared between cohorts using Fisher exact tests. Cumulative incidence of VHD adjusting for the competing risk of death was estimated. ${ }^{11}$ These methods are similar to the Kaplan-Meier method with censoring of patients who are still alive at last follow-up. However, patients who died before experiencing VHD were appropriately accounted for to avoid overestimation of the rate of occurrence of VHD; this can occur if such subjects are simply censored at death. Cox models were used to examine potential associations between baseline factors of interest and the development of VHD. Time-dependent covariates were used to model renal and GI involvement, which developed during follow-up. For all comparisons, a $P$ value $<0.05$ was considered statistically significant. Analyses were performed using SAS version 9.4 (SAS Institute) and R 3.4.2 (R Foundation for Statistical Computing).

\section{RESULTS}

The study included a total of 78 incident SSc cases and 156 non-SSc subjects (mean age 56 [SD 15.7] yrs, 91\% female for both cohorts]. Table 1 summarizes baseline clinical characteristics of the cases and comparators. At index date, patients with SSc had lower prevalence of diabetes ( $3 \%$ vs $12 \% ; P=0.02)$ and obesity $(21 \%$ vs $39 \% ; P=0.008)$ than comparators. The prevalence of pulmonary hypertension (PH) was $8 \%$ in SSc vs $1 \%$ in non-SSc controls $(P=0.003)$. There was no difference in the prevalence of hypertension, hyperlipidemia, or coronary artery disease (CAD).

Table 1. Baseline clinical characteristics.

\begin{tabular}{|c|c|c|c|}
\hline & $\begin{array}{c}\text { SSc, } \\
\mathrm{n}=78\end{array}$ & $\begin{array}{c}\text { Non-SSc, } \\
\mathrm{n}=156\end{array}$ & $P$ \\
\hline Age at diagnosis, yrs & $56.0( \pm 15.6)$ & $56.1( \pm 15.7)$ & 0.97 \\
\hline Female sex & $71(91)$ & $142(91)$ & $>0.99$ \\
\hline Ethnicity & & & 0.24 \\
\hline White & $68(87)$ & $147(94)$ & \\
\hline African American & $2(3)$ & $3(2)$ & \\
\hline Hispanic & $1(1)$ & $2(1)$ & \\
\hline Asian & $5(6)$ & $3(2)$ & \\
\hline Other/unknown & $2(3)$ & $1(1)$ & \\
\hline Follow-up, yrs & \multicolumn{2}{|c|}{$10.5(4.0,17.5) 13.0(6.7,20.0)$} & - \\
\hline $\mathrm{BMI}, \mathrm{kg} / \mathrm{m}^{2}$ & $26.5( \pm 5.9)$ & $29.4( \pm 7.6)$ & 0.004 \\
\hline Obesity (BMI $\geq 30 \mathrm{~kg} / \mathrm{m}^{2}$ ) & $15(21)$ & $61(39)$ & 0.008 \\
\hline Smoking status & & & 0.54 \\
\hline Current & $12(16)$ & $30(20)$ & \\
\hline Former & $24(31)$ & $38(25)$ & \\
\hline 2013 ACR/EULAR ${ }^{a}$ & $71(91)$ & - & - \\
\hline Prior diabetes & $2(3)$ & $19(12)$ & 0.02 \\
\hline Prior hypertension & $30(38)$ & $57(37)$ & 0.77 \\
\hline Prior hyperlipidemia & $27(35)$ & $55(35)$ & 0.92 \\
\hline Prior CAD & $11(14)$ & $17(11)$ & 0.48 \\
\hline Prior PH & $6(8)$ & $1(1)$ & 0.003 \\
\hline Prior ILD & $7(9)$ & - & - \\
\hline \multicolumn{4}{|l|}{ Skin involvement } \\
\hline Limited cutaneous & $65(83)$ & - & \\
\hline Diffuse cutaneous & $11(14)$ & - & \\
\hline Sine scleroderma & $2(3)$ & - & \\
\hline SSc-specific antibodies, $\mathrm{n} / \mathrm{N}(\%)$ & $38 / 75(51)$ & - & \\
\hline Scl70+ & $7 / 35(20)$ & - & \\
\hline $\mathrm{ACA}+$ & $29 / 35(83)$ & - & \\
\hline RNAPIII+ & $2 / 35(6)$ & - & \\
\hline
\end{tabular}

Values are expressed as mean $( \pm S D)$ or median $\left(25^{\text {th }}\right.$ percentile, $75^{\text {th }}$ percentile) for continuous characteristics, and $n(\%)$ or $n / N(\%)$ for discrete characteristics. ${ }^{a}$ Fulfilled 2013 ACR/EULAR classification criteria at baseline. ACA: anticentromere antibody; ACR/EULAR: American College of Rheumatology/European League Against Rheumatism; CAD: coronary artery disease; ILD: interstitial lung disease; $\mathrm{PH}$ : pulmonary hypertension; RNAPIII: RNA polymerase III; SSc: systemic sclerosis. 
Among this cohort of patients, 33 patients with SSc and 31 non-SSc subjects had at least 1 echocardiogram performed before incidence/index date. The prevalence of any VHD at that time was 19/78 (24\%) in patients with SSc vs 8/156 in non-SSc patients $(5 \% ; P<0.001)$. Among the subset of patients who had an echocardiogram, the prevalence of any VHD at that time was $19 / 33(58 \%)$ in patients with SSc vs $8 / 31$ in non-SSc patients $(25 \%, P=0.12)$. Of these patients, 5 with SSc had moderate/ severe VHD (2 moderate/severe mitral regurgitation [MR], 3 moderate/severe tricuspid regurgitation [TR], and 1 moderate/ severe pulmonary regurgitation) vs none in the non-SSc group. There was a higher prevalence of moderate/severe VHD prior to $S S c$ diagnosis compared to non-SSc subjects $(6 \%$ vs $0 \%$; $P=0.004)$. Similarly, among those with echocardiograms, there was a higher prevalence of moderate/severe VHD prior to SSc diagnosis compared to non-SSc subjects ( $15 \%$ vs $0 \% ; P=0.05$ ).

During a median of 10.5 years of follow-up in patients with SSc and 13.0 years of follow-up in non-SSc comparators, 65 patients with SSc and 53 non-SSc subjects had at least 1 echocardiogram performed. Among those without any VHD at SSc incidence/index date, VHD developed during follow-up in 32 patients with SSc and 33 comparators (Table 2). The 10-year cumulative incidence of any VHD during follow-up was higher among patients with SSc $(36.8 \%, 95 \%$ CI 26.1-51.7) compared to non-SSc subjects $(13.2 \%$, 95\% CI 8.5-20.7; $P<0.001)$. Similarly, among patients who received echocardiograms, the 10-year cumulative incidence of any VHD during follow-up was higher among patients with SSc (37.1\%, 95\% CI 23.5-58.7\%) compared to non-SSc subjects $(17.5 \%$, 95\% CI $7.3-42.0 \%$, $P=0.009)$. Of those without moderate/severe VHD at SSc incidence/index date, $18 \mathrm{SSc}$ and 12 non-SSc patients developed moderate/severe VHD (4 aortic stenosis [AS], $3 \mathrm{MR}$, and $14 \mathrm{TR}$ in SSc; $5 \mathrm{AS}, 1$ mitral stenosis, $5 \mathrm{MR}$, and $6 \mathrm{TR}$ in non-SSc). The 10-year cumulative incidence of moderate/ severe VHD during follow-up was higher among patients with SSc (17.9\%, 95\% CI 10.7-29.9) compared to non-SSc subjects $(2.3 \%, 95 \%$ CI $0.7-7.0, P<0.001$; Figure 1). This corresponds to a 4-fold difference (HR 4.23, 95\% CI 2.03-8.83). Similarly, among those with echocardiograms, the 10-year cumulative incidence of moderate/severe VHD was higher among patients with SSc (13.5\%, 95\% CI 5.4-33.4\%) compared to non-SSc subjects
(4.2\%, 95\% CI 0.6-28.4\%), but this difference did not reach statistical significance due to limited sample size $(P=0.22)$. The cumulative incidence for moderate/severe VHD of each type is reported in Table 2.

The prevalence of $\mathrm{PH}$ as cause for moderate/severe TR was 6 $\mathrm{PH}$ (4 probable and 2 definite) in patients with SSc and 1 probable $\mathrm{PH}$ in non-SSc. Secondary TR due to pacemaker leads was not present in our cohort. Supraventricular tachycardia (atrial fibrillation or flutter) adding to TR severity was identified in 7 (9\%) SSc and $9(6 \%)$ non-SSc patients. Preexisting CAD was found to be the only risk factor associated with developing VHD over time in SSc, with 5-fold increased risk (HR 5.66, 95\% CI 1.83-17.51; Table 3). No other traditional CV risk factor such as diabetes, hypertension, smoking status, or dyslipidemia was predictive.

Antiphospholipid antibody (aPL) test results were available in 27/78 SSc cases; none tested positive for lupus anticoagulant; 1 patient with SSc tested positive for anticardiolipin antibody (ACA) and anti- $\beta_{2}$-glycoprotein I, and another one tested positive for ACA alone. Only 1 patient with positive aPL developed moderate/severe valve disease, but none had antiphospholipid syndrome (APS).

\section{DISCUSSION}

To our knowledge, this is the first population-based study to recognize the elevated risk of VHD in SSc, showing up to a 4-fold increased risk of moderate to severe VHD in patients with known SSc. These findings are not limited to secondary tricuspid valve regurgitation in patients with $\mathrm{PH}$ but include dysfunction of the aortic valve (AV) and the mitral valve (MV), leading to stenosis and regurgitation, respectively. AS in particular has a higher-than-expected prevalence and warrants further detailed study.

VHD in SSc has not yet been thoroughly investigated. Most data originate from pathology studies describing endocarditis-like changes on the mitral, tricuspid, or aortic valve in autopsy cases. ${ }^{12}$ Aortic regurgitation and MV prolapse secondary to nodular valve thickening of the $\mathrm{AV}$ and MV have been previously described, but to our knowledge, no systematic study of VHD in a population-based incident cohort has been reported. . $^{13,14,15}$ The most common valvular involvement in SSc is TR, which is mostly, but not solely, secondary related to $\mathrm{PH}$.

Table 2. Cumulative incidence rate of valve disease in 78 patients with SSc compared with 156 subjects without SSc.

\begin{tabular}{lcccc}
\hline Outcome $^{\mathrm{a}}$ & $\begin{array}{c}\text { No. Events After } \\
\text { Incidence/Index } \\
\text { SSc/Non-SSc }\end{array}$ & $\begin{array}{c}\text { Cumulative Incidence at 10 Years, \% } \\
(95 \% \mathrm{CI})^{\mathrm{b}}\end{array}$ & $\begin{array}{c}\text { HR } \\
(95 \% \mathrm{CI})^{\mathrm{c}}\end{array}$ \\
\hline Any valve disease & $32 / 33$ & $36.8(26.1-51.7)$ & $13.2(8.5-20.7)$ & $3.40(2.08-5.57)$ \\
Moderate/severe valve disease & $18 / 12$ & $17.9(10.7-29.9)$ & $2.3(0.7-7.0)$ & $4.23(2.03-8.83)$ \\
Moderate/severe aortic stenosis & $4 / 5$ & $1.4(0.2-9.8)$ & $0.0^{\mathrm{d}}$ & $2.45(0.64-9.45)$ \\
Moderate/severe mitral regurgitation & $3 / 5$ & $2.6(0.7-10.3)$ & $0.8(0.1-5.9)$ & $1.82(0.39-8.42)$ \\
Moderate/severe tricuspid regurgitation & $14 / 6$ & $16.0(9.3-27.6)$ & $1.4(0.4-5.7)$ & $5.73(2.19-14.94)$ \\
\hline
\end{tabular}

a There were no cases of moderate/severe aortic regurgitation, mitral stenosis, tricuspid stenosis, pulmonary stenosis or pulmonary regurgitation in either cohort. ${ }^{b}$ Cumulative incidence is adjusted for the competing risk of death. ${ }^{c}$ Adjusted for age, sex, and calendar year of SSc/index date. ${ }^{\mathrm{d}}$ All events in this group occurred more than 10 years after index date. SSc: systemic sclerosis. 


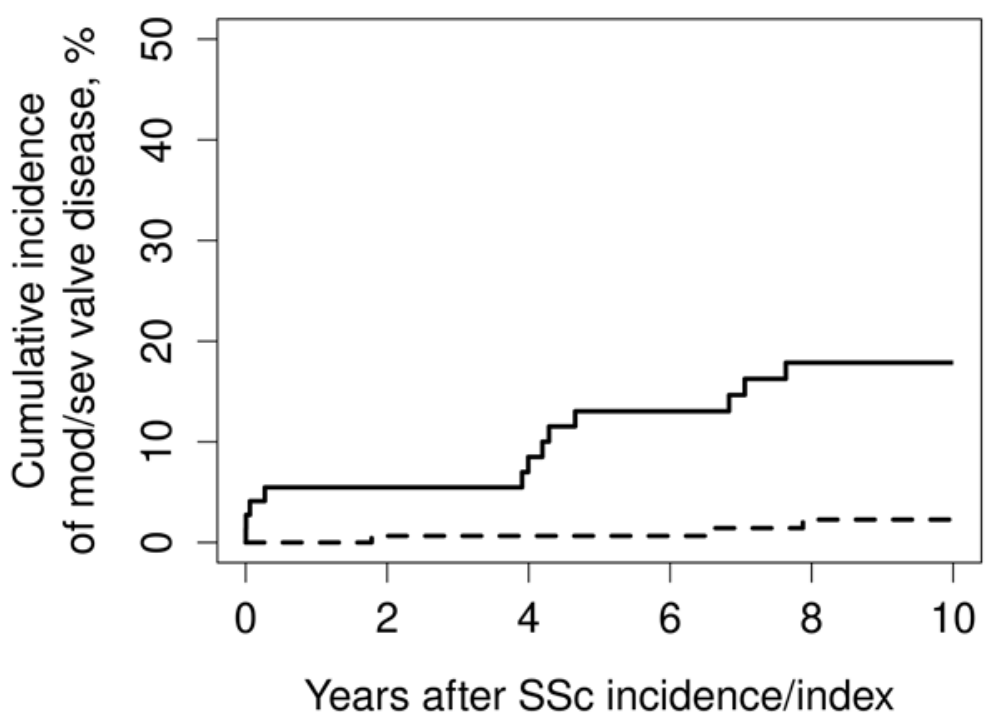

Figure 1. Cumulative incidence of moderate/severe valve disease in patients with SSc (solid line) vs age- and sex-matched non-SSc comparators (dashed line). Mod: moderate; Sev: severe; SSc: systemic sclerosis.

In our cohort, 6/14 (43\%) had secondary TR related to $\mathrm{PH}$. Secondary TR due to pacemaker leads at index/incident date was not present in any SSc or non-SSc comparators. Supraventricular tachycardia (atrial fibrillation or flutter)

Table 3. Risk factors for VHD during follow-up in 73 SSc patients without prior moderate/severe valve disease at SSc diagnosis.

\begin{tabular}{|c|c|c|}
\hline & Mean $( \pm S D)$ or $n(\%)$ & $\begin{array}{c}\text { HR } \\
(95 \% \mathrm{CI})^{\mathrm{a}}\end{array}$ \\
\hline Age, yrs & $55.3( \pm 15.5)$ & $1.36(0.98-1.91)^{\mathrm{b}}$ \\
\hline Sex, male & $6(8)$ & $0.76(0.10-5.76)$ \\
\hline Calendar year of diagnosis & $2001( \pm 9)$ & $1.04(0.97-1.11)$ \\
\hline $\mathrm{CAD}$ at $\mathrm{SSc}$ diagnosis & $8(11)$ & $5.66(1.83-17.51)$ \\
\hline Diabetes & $1(2)$ & - \\
\hline Hypertension & $16(25)$ & $1.28(0.46-3.52)$ \\
\hline Hyperlipidemia & $17(27)$ & $1.11(0.41-3.05)$ \\
\hline Ever smoker & $28(44)$ & $1.76(0.69-4.46)$ \\
\hline Current smoker & $10(16)$ & $1.09(0.24-4.86)$ \\
\hline $\mathrm{BMI}, \mathrm{kg} / \mathrm{m}^{2}$ & $26.0( \pm 5.9)$ & $0.99(0.90-1.08)$ \\
\hline Obesity $\left(\mathrm{BMI} \geq 30 \mathrm{~kg} / \mathrm{m}^{2}\right)$ & $11(19)$ & $1.25(0.39-3.96)$ \\
\hline Telangiectasia & $35(48)$ & $0.82(0.30-2.21)$ \\
\hline Digital ulcers & $14 / 29(48)$ & $0.39(0.08-1.93)$ \\
\hline Pulmonary artery hypertension & $2(3)$ & $3.41(0.42-28.04)$ \\
\hline Interstitial lung disease & $6(8)$ & $0.56(0.06-5.11)$ \\
\hline $\mathrm{RP}$ & $70(96)$ & $0.31(0.04-2.47)$ \\
\hline Diffuse vs limited/sine & $11(15)$ & $1.50(0.42-5.39)$ \\
\hline Calcinosis & $15(21)$ & $1.37(0.50-3.74)$ \\
\hline GI involvement ${ }^{c}$ & 39 & $1.86(0.69-5.02)$ \\
\hline Renal involvement ${ }^{\mathrm{c}}$ & 6 & $1.07(0.14-8.10)$ \\
\hline $\mathrm{ACA}+$ & $26 / 35(74)$ & $0.66(0.16-2.64)$ \\
\hline Anti-Scl70 + & $7 / 35(20)$ & $0.90(0.18-4.42)$ \\
\hline
\end{tabular}

${ }^{\mathrm{a}}$ Age-adjusted univariable models. ${ }^{\mathrm{b}}$ Per 10 years. ${ }^{\mathrm{c}}$ Time-dependent variable. ACA: anticentromere antibody; CAD: coronary artery disease; GI: gastrointestinal; RP: Raynaud phenomenon; SSc: systemic sclerosis; VHD: valvular heart disease. adding to TR severity in patients with $S S c$ was identified in 7 (9\%) and $9(6 \%)$ non-SSc patients at incidence/index date. Nevertheless, the majority had primary tricuspid valve changes leading to moderate/severe TR.

Interestingly, patients with SSc seem to develop VHD prematurely. The VHD that was seen in our study occurred in a much younger population than in the general non-SSc population. ${ }^{16}$ Chronic inflammation is known to be associated with accelerated atherosclerosis and has also been associated with myocarditis and cardiomyopathies. ${ }^{17}$ Inflammation may have a role in VHD development and progression, especially AS, as seen in patients with rheumatoid arthritis and psoriasis. ${ }^{18,19}$ In SSc, microvascular dysfunction may potentially lead to earlier fibrotic changes in the cardiac valves. Nevertheless, there is no study that has specifically evaluated systematically the pathologic changes in VHD in autoimmune rheumatologic disorders. In the general population, there appear to be sex-based differences in mechanisms leading to VHD. In females, valvular fibrosis is more prominent than valvular calcification in severe AS. ${ }^{20,21}$ Therefore hypothetically in $S \mathrm{Sc}$, which has a female predominance, a higher prevalence of valvular pathology may be secondary to the autoimmunity, which hastens fibrotic tissue changes. In addition, the increased prevalence of VHD before SSc diagnosis suggests that subclinical disease activity may alter cardiac structures such as heart valves even before cutaneous or other organ manifestations become apparent. Valvular pathology has been more extensively described in systemic lupus erythematosus (SLE). Typical valvular lesions in SLE have been described as nodules or nonbacterial thrombotic endocarditis (ranging from small lesions to large verrucous masses), often related to APS. ${ }^{22}$ There were no patients with APS in our SSc cohort. Other chronic inflammatory diseases such as psoriasis show an increased risk of developing AS not explained by traditional CV risk factors. ${ }^{18}$ Case studies have described endocarditis-like changes in patients 
with SSc, but there are no prior studies on the prevalence of VHD in this population of patients. ${ }^{23,24}$

The primary strengths of our study include the use of the REP, a population-based cohort using a comprehensive record-linkage system able to capture information on the health care of all residents of Olmsted County regardless of age, sex, ethnicity, socioeconomic status, insurance status, or setting of healthcare delivery. By using this system, we were able to follow SSc cases across the full spectrum of disease for decades, from symptoms through final diagnosis, without relying only on administrative data, unlike referral cohorts. By manually reviewing every medical record, we were able to confirm diagnosis and minimize the eventuality of disease misdiagnosis, which is a major concern in studies that are based on administrative claims data.

The development of VHD takes multiple years; therefore, following patients for an extended time is crucial to detect changes in valve function. Finally, the population is relatively stable, so the duration of medical record information available to investigators was substantial.

The limitations include the retrospective design of our study and the limited numbers of observed, incident SSc patients. Due to the design, clinical and echocardiography information was not collected prospectively. Patients without an echocardiogram were rated as not having VHD. This might be valid for moderate and severe VHD but is prone to underestimation of mild valvular disease. Surveillance bias might contribute significantly in SSc studies, due to the frequent and much closer monitoring of these patients for PH. Patients with SSc undergo more frequent clinical and echocardiographic examinations, as well as laboratory testing.

SSc is a disease with a high female predominance. Sex differences in CV risk factors and VHD have been evaluated in other studies. ${ }^{25,26,27}$ Nevertheless, sex difference in VHD in patients with SSc is unknown and difficult to elucidate.

This study was conducted in Olmsted County, Minnesota, where the predominant population is White. Some ethnic and racial groups are underrepresented in our population. Therefore, the generalizability of this study to other ethnic cohorts might vary, and our results must be looked at on a case-by-case basis when generalizing to more diverse populations..$^{28}$

In conclusion, although VHD has not been considered to be a major primary cardiac manifestation of SSc, our current study revealed a higher-than-anticipated prevalence and incidence of valvular involvement in these patients compared to age- and sex-matched comparators without SSc. Persons with SSc have a 4-fold increased prevalence of moderate/severe VHD at diagnosis compared to persons with non-SSc. They also have a 4 -fold increased risk of developing moderate/severe valvular dysfunction after diagnosis of SSc compared to non-SSc subjects. AV and MV disease is of key interest and cannot be explained by ILD or PH, which are often associated with secondary pulmonary or tricuspid valve pathology in these patients. Underlying mechanisms for this association require further study. SSc-associated cardiac disease is linked with major increase in morbidity and mortality; therefore, screening with appropriate diagnostic tools is essential for early detection. The optimal evaluation and management of cardiac involvement in patients with SSc require an integrated multidisciplinary approach involving rheumatologists, pulmonologists, and cardiologists.

\section{DATA SHARING}

Data are available upon reasonable request. Data requests should be sent to AM.

\section{REFERENCES}

1. Denton CP, Black CM, Korn JH, de Crombrugghe B. Systemic sclerosis: current pathogenetic concepts and future prospects for targeted therapy. Lancet 1996;347:1453-8.

2. Allanore Y, Simms R, Distler O, Trojanowska M, Pope J, Denton CP, et al. Systemic sclerosis. Nat Rev Dis Primers 2015;1:15002.

3. Bissell LA, Anderson M, Burgess M, Chakravarty K, Coghlan G, Dumitru RB, et al. Consensus best practice pathway of the UK systemic sclerosis study group: management of cardiac disease in systemic sclerosis. Rheumatology 2017;56:912-21.

4. Tyndall AJ, Bannert B, Vonk M, Airo P, Cozzi F, Carreira PE, et al. Causes and risk factors for death in systemic sclerosis: a study from the EULAR Scleroderma Trials and Research (EUSTAR) database. Ann Rheum Dis 2010;69:1809-15.

5. Desai CS, Lee DC, Shah SJ. Systemic sclerosis and the heart: current diagnosis and management. Curr Opin Rheumatol 2011;23:545-54.

6. Parks JL, Taylor MH, Parks LP, Silver RM. Systemic sclerosis and the heart. Rheum Dis Clin North Am 2014; 40:87-102.

7. Papagoras C, Achenbach K, Tsifetaki N, Tsiouris S, Fotopoulos A, Drosos AA. Heart involvement in systemic sclerosis: a combined echocardiographic and scintigraphic study. Clin Rheumatol 2014;33:1105-11.

8. van den Hoogen F, Khanna D, Fransen J, Johnson SR, Baron M, Tyndall A, et al. 2013 classification criteria for systemic sclerosis: an American College of Rheumatology/European League Against Rheumatism collaborative initiative. Ann Rheum Dis 2013;72:1747-55.

9. St Sauver JL, Grossardt BR, Yawn BP, Melton LJ 3rd, Rocca WA. Use of a medical records linkage system to enumerate a dynamic population over time: the Rochester Epidemiology Project. Am J Epidemiol 2011;173:1059-68.

10. Nishimura RA, Otto CM, Bonow RO, Carabello BA, Erwin JP 3rd, Guyton RA, et al; ACC/AHA Task Force Members. 2014 AHA/ ACC guideline for the management of patients with valvular heart disease: executive summary: a report of the American College of Cardiology/American Heart Association task force on practice guidelines. Circulation 2014;129:2440-92.

11. Gooley TA, Leisenring W, Crowley J, Storer BE. Estimation of failure probabilities in the presence of competing risks: new representations of old estimators. Stat Med 1999;18:695-706.

12. Oram $S$, Stokes W. The heart in scleroderma. Br Heart J 1961;23:243-59.

13. Lambova S. Cardiac manifestations in systemic sclerosis. World J Cardiol 2014;6:993-1005.

14. D’Angelo WA, Fries JF, Masi AT, Shulman LE. Pathologic observations in systemic sclerosis (scleroderma). A study of fifty-eight autopsy cases and fifty-eight matched controls. Am J Med 1969;46:428-40.

15. Champion HC. The heart in scleroderma. Rheum Dis Clin North Am 2008;34:181-90.

16. Nkomo VT, Gardin JM, Skelton TN, Gottdiener JS, Scott CG, Enriquez-Sarano M. Burden of valvular heart diseases: a population-based study. Lancet 2006;368:1005-11. 
17. Voskuyl AE. The heart and cardiovascular manifestations in rheumatoid arthritis. Rheumatology 2006;45:iv4-7.

18. Khalid U, Ahlehoff O, Gislason GH, Skov L, Torp-Pedersen C, Hansen PR. Increased risk of aortic valve stenosis in patients with psoriasis: a nationwide cohort study. Eur Heart J 2015;36:2177-83.

19. Bois JP, Crowson CS, Khullar T, Achenbach SJ, Krause ML, Mankad R. Progression rate of severity of aortic stenosis in patients with rheumatoid arthritis. Echocardiography 2017;34:1410-6.

20. Thaden JJ, Nkomo VT, Suri RM, Maleszewski JJ, Soderberg DJ, Clavel MA, et al. Sex-related differences in calcific aortic stenosis: correlating clinical and echocardiographic characteristics and computed tomography aortic valve calcium score to excised aortic valve weight. Eur Heart J 2016;37:693-9.

21. Aggarwal SR, Clavel MA, Messika-Zeitoun D, Cueff C, Malouf J, Araoz PA, et al. Sex differences in aortic valve calcification measured by multidetector computed tomography in aortic stenosis. Circ Cardiovasc Imaging 2013;6:40-7.

22. Khamashta MA, Cervera R, Asherson RA, Font J, Gil A, Coltart DJ, et al. Association of antibodies against phospholipids with heart valve disease in systemic lupus erythematosus. Lancet 1990;335:1541-4.
23. De Langhe E, Seghers A, Demaerel P, Verschueren P, Lemmens R. Non-infective endocarditis with systemic embolization and recurrent stroke in systemic sclerosis. Rheumatology 2015; 55:589-91.

24. Roth LM, Kissane JM. Panaortitis and aortic valvulitis in progressive systemic sclerosis (scleroderma). Am J Clin Pathol 1964;41:287-96.

25. Porras AM, McCoy CM, Masters KS. Calcific aortic valve disease: a battle of the sexes. Circ Res 2017;120:604-6.

26. Low TT, Chan SP, Wai SH, Ang Z, Kyu K, Lee KY, et al. The women's heart health programme: a pilot trial of sex-specific cardiovascular management. BMC Womens Health 2018;18:56.

27. Anand SS, Islam S, Rosengren A, Franzosi MG, Steyn K, Yusufali AH, et al; INTERHEART Investigators. Risk factors for myocardial infarction in women and men: insights from the INTERHEART study. Eur Heart J 2008;29:932-40.

28. St Sauver JL, Grossardt BR, Leibson CL, Yawn BP, Melton LJ 3rd, Rocca WA. Generalizability of epidemiological findings and public health decisions: an illustration from the Rochester Epidemiology Project. Mayo Clin Proc 2012;87:151-60. 